\title{
COMPETENZE DELL'INFERMIERE IN RELAZIONE AL SERVIZIO MOBILE DI EMERGENZA - SAMU
}

\section{RECENSIONE INTEGRATIVA}

REIS, Adilson dos ${ }^{1}$

EVARISTO, Leidiane da Silva ${ }^{2}$

SOUSA, Clarisse Cristina Ferreira Silva ${ }^{3}$

CASTRO, Mônica França de ${ }^{4}$

GUASTALDI, Rosimeire ${ }^{5}$

REIS, Adilson dos. Et al. Competenze dell'infermiere in relazione al servizio mobile di emergenza - SAMU. Revista Científica Multidisciplinar Núcleo do Conhecimento. anno 04, Ed. 11, Vol. 04, pp. 159-170. novembre 2019. ISSN: 24480959, Collegamento

accesso: https://www.nucleodoconhecimento.com.br/salute/competenze-infermiere

\section{RIEPILOGO}

Introduzione: In Brasile, le reti di emergenza e di emergenza sono supportate e regolamentate dal sistema sanitario unificato, il professionista infermieristico è essenziale per le pratiche di assistenza svolte dai servizi di assistenza mobile di emergenza (SAMU), non solo per quanto riguarda l'orientamento e il supporto medico,

\footnotetext{
${ }^{1}$ Laurea in Infermieristica.

${ }^{2}$ Laureato in Infermieristica.

${ }^{3}$ Laureato in Infermieristica.

${ }^{4}$ Laureato in Infermieristica.

${ }^{5}$ Dottorato in corso in Scienze della Salute. Laurea magistrale in Scienze della Salute. Specializzazione nella gestione dei servizi infermieristici. Infermieristica universitaria.
} 
si nota che l'assistenza infermieristica ha un gran numero di funzioni relative alla sua assistenza in SAMU. Obiettivi: Identificare il lavoro degli infermieri in relazione al servizio di assistenza di emergenza mobile. Metodo: È stata condotta una revisione sistematica della letteratura, con l'analisi delle principali riviste infermieristiche nelle banche dati Scientific Electronic Library Online (SciELO), nella letteratura latinoamericana e caraibica nelle scienze della salute (Lilacs) e nel sistema online di ricerca e analisi della letteratura medica (Pubmed/MEDLINE). Un totale di 47 articoli sono stati raccolti con il tema dello studio. Risultati: È stato dimostrato che vi è una notevole quantità di articoli relativi alle competenze dell'infermiere nei confronti della SAMU e alla rilevanza della professione per il corretto funzionamento della SAMU. Conclusione: $\mathrm{Si}$ conclude che attraverso lo sviluppo scientifico e tecnico della professione, con la realizzazione di corsi di laurea e di laurea, le attribuzioni infermieristiche in relazione alla SAMU sono considerate importanti, ma che ulteriori ricerche sono ancora necessarie per studenti di questa categoria.

Parole chiave: Infermieristica, Mobile Emergency Care Service, incarichi infermieristici.

\section{INTRODUZIONE}

In Brasile, le reti di emergenza e di emergenza sono supportate e regolate dal sistema sanitario unificato, ordinanza $n$ 1.600, 2011. E sono stabiliti dai segmenti di: promozione della salute, prevenzione e sorveglianza, oltre ai servizi mobili di assistenza di emergenza (SAMU). Gli infermieri sono i professionisti con formazione scientifica e competenza umana per affrontare la vita, e il loro ruolo è di fondamentale importanza per l'assistenza che richiede procedure invasive nei casi che coinvolgono unità di supporto vitale avanzate (VAS). ${ }^{1}$

L'assistenza infermieristica è un'ampia scienza che contempla i diritti umani e la salute, con uno sguardo umanizzato rispetto allo sviluppo umano e conquista sempre più il suo spazio nei settori sanitari più diversi. In passato, l'infermiere era visto come un professionista ausiliario del medico, oggi si comprende questa professione con ampiezza, che ha diversi contributi che favoriscono i pazienti, sia in materia di informazione, procedura, gestione attrezzature e umanizzazione. ${ }^{2}$ 
Il professionista infermieristico è essenziale per le pratiche di cura svolte da SAMU, non solo per quanto riguarda l'orientamento e il supporto medico, ma anche per l'assistenza umile, con una vasta visione di organizzazione, gestione del team, ha incontrato attrezzature e manovre che salvano vite umane. Pertanto, è possibile che gli infermieri si concentrino costantemente sull'istruzione continua, in modo che diventi sempre più specializzata nella fornitura di Primo Soccorso, per sviluppare il loro lavoro con qualità ed efficacia. ${ }^{3}$

Secondo Costa 2016, le prestazioni dell'infermiere nell'assistenza mobile preospedaliera dovrebbero essere ampliate, perché è un campo estremamente importante per la vita e più efficiente è questa cura, maggiore è la possibilità di vita di un individuo. Di conseguenza, per Mendes 2010, il Mobile Emergency Care Service SAMU rappresenta un'assistenza cruciale per l'individuo che attraversa alcune complicazioni sanitarie e deve essere assistito da professionisti in forma e in grado di eseguire tutti i procedure necessarie, con l'obiettivo di conservare la vita ${ }^{3}$

La qualità dei servizi sanitari offerti in Brasile negli anni ' 80 ha subito cambiamenti significativi, spinti dalla mancanza di risorse finanziarie che hanno sottolineato l'importanza di adottare un nuovo modo di gestire e gestire le risorse finanziarie. La gestione della qualità fatta dall'assistenza infermieristica rappresenta una grande importanza, quindi è intesa come una strategia che raggiunge un accettabile standard continuo di assistenza di qualità. La gestione consente lo sviluppo di risorse umane generando responsabilità per il team, inclusi manager e amministratori. ${ }^{4}$

Riconoscendo che gli infermieri sono indispensabili nell'assistenza pre-ospedaliera, diventa di grande interesse comprendere le competenze e i contributi al settore, sorge la domanda: quali sono le attribuzioni dell'infermiere in relazione all'assistenza nel Samu?

\section{QUADRO TEORICO}

L'elevata domanda di servizi di assistenza di emergenza efficaci e di emergenze mediche ha generato diversi modelli di sistemi di assistenza in tutto il mondo. In 
Brasile, la prima proposta ha avuto luogo nel 2003, attraverso la politica nazionale di attenzione alle emergenze - (PNAU) ${ }^{5}$

Secondo Mendes 2010, questa politica rappresenta lo sforzo del Ministero della Salute per organizzare i flussi regionali di assistenza globale per i casi di urgenza e di emergenza in Brasile. Così, il PNAU è guidato dai principi del SUS ed è strutturato in cinque assi, questi: promuovere la qualità della vita; networking; funzionamento degli impianti di regolamentazione; formazione e formazione continua; umanizzazione dell'attenzione.6

La sua attuazione è stata caratterizzata dalla componente mobile pre-ospedaliera, presentandosi come una strategia nel Mobile Emergency Care Service (SAMU), che prevede un modello standardizzato di assistenza, in cui l'assistenza viene fornita dopo una chiamata gratuitamente per il numero 192, e il centro medico definisce come devono essere condotte le cure ${ }^{6}$

Inoltre, si è verificato un ampliamento dell'accesso ai servizi, non solo attraverso il servizio fornito da SAMU, nonché l'attuazione delle unità di emergenza (UPA), che si presentano come la più recente strategia della politica, unendo la strategia di formazione e formazione continua dei professionisti, chiamati Nuclei e Istruzione e Urgenza. ${ }^{6}$

\section{RISULTATI}

Cercando di comprendere l'importanza degli infermieri in relazione al servizio mobile di emergenza (SAMU), durante la raccolta dei dati, tenendo conto dei criteri di inclusione ed esclusione, sono stati trovati 9 articoli scientifici che affrontavano le competenze cura infermieri e sette manageriali nelle pubblicazioni presentate nelle tabelle nel precedente articolo, in due fasi, la prima dice circa la caratterizzazione degli articoli pubblicati riguardanti il titolo, il luogo, l'autore, l'anno, il tipo di studio e l'obiettivo e il secondo per quanto riguarda il competenze, attività e percentuali correlate. 
La raccolta dei dati di questa revisione integrativa è stata effettuata dalla selezione degli studi descritti nella Tabella 1

Tabella 1 - Caratterizzazione degli articoli pubblicati riguardanti titolo, luogo, autore, anno, tipo di studio e obiettivo. São Paulo, 2019

\begin{tabular}{|c|c|c|c|c|}
\hline $\begin{array}{l}\text { Identificazion } \\
\mathrm{e}\end{array}$ & Titolo, autore & $\begin{array}{l}\text { Ubicazione, } \\
\text { anno }\end{array}$ & $\begin{array}{ll}\text { Tipo } & \text { di } \\
\text { studio } & \end{array}$ & Obiettivo \\
\hline A1 & $\begin{array}{l}\text { Infermieri del servizio } \\
\text { di assistenza mobile } \\
\text { di emergenza: profilo } \\
\text { e attività sviluppate. } \\
\text { Luchtemberg MN, } \\
\text { Pires DEP }\end{array}$ & $\begin{array}{l}\text { Florianópoli } \\
\text { s, } 2015\end{array}$ & $\begin{array}{l}\text { Studio } \\
\text { descrittivo } \\
\text { qualitativo }\end{array}$ & \begin{tabular}{l}
\multicolumn{2}{l}{ Caratterizzare il } \\
profilo \\
identificare le \\
attività \\
sviluppate dagli \\
infermieri dal \\
servizio di \\
assistenza \\
mobile di \\
emergenza di \\
uno stato nel \\
sud del Brasile
\end{tabular} \\
\hline A2 & $\begin{array}{l}\text { Continuare la } \\
\text { formazione continua } \\
\text { come strategie di } \\
\text { gestione nel servizio } \\
\text { di assistenza mobile } \\
\text { di emergenza. Hetti } \\
\text { LBE, Bernardes A, } \\
\text { Gabriel CS, Fortuna } \\
\text { CM, Maziero VG. }\end{array}$ & $\begin{array}{l}\text { São Paulo, } \\
2013\end{array}$ & $\begin{array}{l}\text { Studio } \\
\text { qualitativo }\end{array}$ & $\begin{array}{l}\text { Lo studio } \\
\text { mirava ad } \\
\text { analizzare la } \\
\text { percezione dei } \\
\text { professionisti } \\
\text { sulla } \\
\text { formazione } \\
\text { permanente e/o } \\
\text { continua nel } \\
\text { Servizio Mobile } \\
\text { di Emergenza } \\
\text { di un Comune }\end{array}$ \\
\hline
\end{tabular}




\begin{tabular}{|c|c|c|c|c|}
\hline & & & & $\begin{array}{l}\text { dello Stato di } \\
\text { San Paolo }\end{array}$ \\
\hline A3 & $\begin{array}{l}\text { Il punto di vista } \\
\text { dell'infermiere/manag } \\
\text { er sulla necessità di } \\
\text { implementare il } \\
\text { supporto psicologico } \\
\text { ai professionisti del } \\
\text { servizio di assistenza } \\
\text { mobile di emergenza; } \\
\text { Meskita KL, Gomes } \\
\text { GPLA, Silva MJBF, } \\
\text { Santos LF. }\end{array}$ & $\begin{array}{l}\text { Tocantins, } \\
2014 .\end{array}$ & $\begin{array}{l}\text { Ricerca } \\
\text { qualitativa } \\
\text { descrittiva } \\
\text { e } \\
\text { esplorativa }\end{array}$ & $\begin{array}{l}\text { Analizzare il } \\
\text { punto di vista } \\
\text { dell'infermiera/g } \\
\text { e sulla } \\
\text { necessità di } \\
\text { implementare il } \\
\text { supporto } \\
\text { psicologico per i } \\
\text { professionisti } \\
\text { SAMU }\end{array}$ \\
\hline A4 & $\begin{array}{l}\text { La vita quotidiana } \\
\text { degli infermieri che } \\
\text { lavorano nel servizio } \\
\text { di assistenza mobile } \\
\text { di emergenza. } \\
\text { Tavares TY, Santana } \\
\text { JCB, Eloy MD, } \\
\text { Oliveira RD, Paula RF }\end{array}$ & $\begin{array}{l}\text { Minas } \\
\text { Gerais, } \\
2017\end{array}$ & $\begin{array}{l}\text { Ricerca } \\
\text { qualitativa } \\
\text { ed } \\
\text { esplorativa }\end{array}$ & $\begin{array}{l}\text { Comprendere il } \\
\text { lavoro } \\
\text { quotidiano degli } \\
\text { infermieri che } \\
\text { lavorano nel } \\
\text { servizio mobile } \\
\text { di emergenza }\end{array}$ \\
\hline A5 & $\begin{array}{l}\text { Prospettive degli } \\
\text { infermieri in cura } \\
\text { nell'assistenza mobile } \\
\text { pre-ospedaliera. } \\
\text { Anjos MS, Oliveira } \\
\text { SS, Rosa DOS }\end{array}$ & Bahia, 2016 & $\begin{array}{l}\text { Studio } \\
\text { esplorativo } \\
\text { qualitativo }\end{array}$ & $\begin{array}{l}\text { Descrivere le } \\
\text { prospettive } \\
\text { degli infermieri } \\
\text { in cura } \\
\text { nell'assistenza } \\
\text { mobile pre- } \\
\text { ospedaliera. }\end{array}$ \\
\hline A6 & $\begin{array}{l}\text { Prestazioni degli } \\
\text { infermieri } \\
\text { nell'assistenza mobile }\end{array}$ & $\begin{array}{l}\text { São Paulo, } \\
2013\end{array}$ & $\begin{array}{l}\text { Revisione } \\
\text { bibliografic }\end{array}$ & $\begin{array}{l}\text { L'obiettivo di } \\
\text { questo studio } \\
\text { era quello di }\end{array}$ \\
\hline
\end{tabular}




\begin{tabular}{|c|c|c|c|c|}
\hline & $\begin{array}{l}\text { pre-ospedaliera. } \\
\text { Adão RS, Santos MR. }\end{array}$ & & $\begin{array}{l}\text { a } \\
\text { qualitativa }\end{array}$ & $\begin{array}{l}\text { descrivere le } \\
\text { azioni degli } \\
\text { infermieri in } \\
\text { un'unità } \\
\text { sanitaria di } \\
\text { base } \\
\text { avanzata nel } \\
\text { PHA mobile, } \\
\text { attraverso la } \\
\text { revisione } \\
\text { letteraria. }\end{array}$ \\
\hline A7 & $\begin{array}{l}\text { L'esecuzione } \\
\text { dell'infermiera di un } \\
\text { servizio di assistenza } \\
\text { pre-ospedaliera } \\
\text { privata. Peres PSQ, } \\
\text { Arboit } \\
\text { Camponogara S, } \\
\text { Pilau COB, Menezes } \\
\text { OS, Kaefer CT. }\end{array}$ & $\begin{array}{l}\text { Rio Grande } \\
\text { do Sul, } 2018\end{array}$ & $\begin{array}{l}\text { Studio } \\
\text { descrittivo } \\
\text { esplorativo } \\
\text { qualitativo }\end{array}$ & $\begin{array}{l}\text { Conoscere la } \\
\text { percezione } \\
\text { degli operatori } \\
\text { sanitari circa le } \\
\text { prestazioni } \\
\text { dell'infermiere } \\
\text { in un servizio di } \\
\text { assistenza pre- } \\
\text { ospedaliera. }\end{array}$ \\
\hline A8 & $\begin{array}{l}\text { Prestazioni degli } \\
\text { infermieri } \\
\text { nell'assistenza mobile } \\
\text { pre-ospedaliera. } \\
\text { Fernandez MG, } \\
\text { Espindula BM. }\end{array}$ & $\begin{array}{l}\text { São Paulo, } \\
2018\end{array}$ & $\begin{array}{l}\text { Revisione } \\
\text { integrativa } \\
\text { della } \\
\text { letteratura }\end{array}$ & $\begin{array}{l}\text { L'obiettivo di } \\
\text { questo studio } \\
\text { era quello di } \\
\text { descrivere le } \\
\text { azioni degli } \\
\text { infermieri in } \\
\text { un'unità } \\
\text { sanitaria } \\
\text { base di } \\
\text { avanzata nel } \\
\text { PHA mobile, }\end{array}$ \\
\hline
\end{tabular}




\begin{tabular}{|c|c|c|c|c|}
\hline & & & & $\begin{array}{l}\text { attraverso la } \\
\text { revisione } \\
\text { letteraria. }\end{array}$ \\
\hline A9 & $\begin{array}{l}\text { Supervisione delle } \\
\text { infermiere } \\
\text { nell'assistenza mobile } \\
\text { pre-ospedaliera. } \\
\text { Bernardes A, Maziero } \\
\text { GV, Hetti LBE, Baldin } \\
\text { MSC, Gabriel CS. }\end{array}$ & $\begin{array}{l}\text { São Paulo, } \\
2014\end{array}$ & $\begin{array}{l}\text { Ricerca } \\
\text { descrittiva } \\
\text { qualitativa }\end{array}$ & $\begin{array}{l}\text { Lo studio } \\
\text { mirava ad } \\
\text { analizzare } \\
\text { come avviene } \\
\text { la supervisione } \\
\text { nel Servizio } \\
\text { Mobile di } \\
\text { Emergenza } \\
\text { (SAMU) di un } \\
\text { comune dello } \\
\text { Stato di San } \\
\text { Paolo }\end{array}$ \\
\hline
\end{tabular}

Fonte: Kings, 2019.

Gli studi selezionati sono stati analizzati e raggruppati in base alle competenze trovate, attività e percentuale, descritte nel grafico 2 .

Tabella 2: Distribuzione degli articoli trovati in merito alle competenze di cura e di formazione manageriale. São Paulo, 2019

\begin{tabular}{|c|c|c|c|}
\hline digitare & Articoli & Attività & $\%$ \\
\hline Assistenza & $\begin{array}{l}\text { A1 } \\
\text { A4 } \\
\text { A5 } \\
\text { A6 } \\
\text { A7 } \\
\text { A8 }\end{array}$ & $\begin{array}{l}\text { - Valutazione delle lesioni e trattamento; } \\
\text { - Farmaci } \quad \text { come prescrizione; } \\
\text { - Foratura ad accesso venoso, sonda vescica e } \\
\text { nasogastrico; } \\
\text { - Monitoraggio cardiaco e ossimetria a impulsi; } \\
\text { - Valutazione emodinamica, cura della ventilazione } \\
\text { meccanica; } \\
\text { - Condimenti; }\end{array}$ & $66,6 \%$ \\
\hline
\end{tabular}




\begin{tabular}{|c|c|c|c|}
\hline Gestione & $\begin{array}{l}\text { A2 } \\
\text { A3 } \\
\text { A9 }\end{array}$ & $\begin{array}{l}\text { - Attività di gestione e/o attività amministrative; } \\
\text { - Condurre formazione e istruzione permanente; } \\
\text { - Protocolli di costruzione; } \\
\text { - Supervisionare e valutare le azioni del team in APH } \\
\text { mobile; } \\
\text { - Completamento del foglio del caso; } \\
\text { - Controllare l'elenco dei materiali, dei medicinali e } \\
\text { delle } \\
\text { - Report in un verbale contabile, inventario della base } \\
\text { e controllo dell'inventario, rapporto infermieristico, } \\
\text { richiesta di medicinali e materiali; } \\
\text { - Disinfezione di attrezzature e veicoli; } \\
\text { - forma di droga controllata, termine di responsabilità } \\
\text { di trasferimento, controllo della temperatura del } \\
\text { magazzino; } \\
\text { - Scala di disinfezione del veicolo, riempimento di } \\
\text { cartelle cliniche, partecipazione all'elaborazione di } \\
\text { progetti come le educazioni samu; } \\
\text { - Partecipazione alle riunioni e compilazione del } \\
\text { foglio di lavoro }\end{array}$ & \\
\hline Totale: & & & \\
\hline
\end{tabular}

Fonte: Kings, 2019

\section{DISCUSSIONE}

Per comprendere meglio i risultati, saranno discussi in due argomenti: competenze di cura per le prestazioni dell'infermiere nella SAMU e capacità di gestione per il ruolo dell'infermiere nella SAMU. 


\section{CARE SKILLSE DI NURSES A ACT IN SAMU}

Le pubblicazioni si sono rivolte contemporaneamente alle competenze dell'infermiere, sia di cura che di gestione, ma del $33 \%$ (n. 3) delle pubblicazioni, l'obiettivo principale è stato quello dell'infermiere come manager, tra le competenze trovate, quelle che si distinguono maggiormente e sono ricorrenti sono: la valutazione dell'estensione delle malattie dando priorità a un maggiore impegno e all'esecuzione delle procedure e delle cure. Spiccatrando tra le procedure, la foratura venosa, il passaggio del tubo di taglio della vescica e la sonda nasogastrica, mentre nella fornitura di cure è stato trovato: prestazioni di medicazioni, monitoraggio dei segni vitali e somministrazione di farmaci, monitoraggio cardiaco e ossimetria cardiaca, igiene e comfort, valutazione emodinamica, immobilizzazioni, anamnesi, assistenza medica in procedure come la rianimazione e il processo decisionale. ${ }^{6}$

Tra gli altri. Date le molteplici funzioni svolte dall'infermiere, è chiaro l'idea dell'importanza dell'infermiere e della responsabilità che gli è stata affidata, che richiede lo sviluppo di competenze che coinvolgano la cognizione, gli atteggiamenti e l'esecuzione delle procedure, che termina per ruolo di manager, il che significa in molti casi l'emergere di esaurimento fisico e mentale. Pertanto, è essenziale fornire risorse umane e fisiche al fine di fornire la migliore cura alla vittima, sempre mirando a ridurre al minimo i problemi.

\section{MANAGERIAL SKILLSE DI NURSES A WORK IN SAMU}

Per le principali competenze gestionali, è stato dimostrato che, è compito degli infermieri sviluppare protocolli per la corretta fornitura di assistenza, considerando tutto il campione che giustifica la cura e l'impegno del paziente.8 Inoltre, con la cura e l'impegno del paziente infermiere, il completamento del modulo di presenza, il controllo e il rifornimento dello stock di materiali che devono essere nel veicolo e che sono rilevanti per l'assistenza, la disinfezione di attrezzature e veicoli, il rapporto infermieristico, la partecipazione o sviluppo di progetti che consentano l'educazione sanitaria in modo permanente ${ }^{9}$ 
Data l'evidenza delle competenze degli infermieri, si comprende l'importanza e che questo professionista è indispensabile considerando sia le attività di assistenza e di gestione, che sono private dell'infermiere, sia per fornire sostegno ai collaboratori del team infermieristico, che porta all'indice di qualità della consegna dell'assistenza, capire che la non prestazione, la non conoscenza delle loro attività rilevanti può caratterizzare i danni o la morte per la vittima assistita. ${ }^{9}$

Puntando al di là di tutto, la buona qualità dell'assistenza infermieristica è custoly fino all'infermiera per gestire il team infermieristico, e lo stesso dovrebbe essere responsabile per le dimensioni del team, considerando le prestazioni di ogni dipendente, l'elaborazione protocolli che si basano su prove scientifiche e adattati alla realtà della salute brasiliana, forniscono modi per eseguire le cure e che sostengono le azioni del professionis ${ }^{\text {ta.10 }}$ Secondo Santos e Adam, 2013 l'introduzione del professionista Nurse in care pre-ospedale mobile è stato dato attraverso il PNAU, che entra in accordo con quanto dice il Consiglio Regionale infermieristico, che considera questo importante professionale per la composizione del team PHA, dal momento che è in grado di sviluppare azioni con l'obiettivo di preservare e ridurre i danni alla vita, sia attraverso l'assistenza diretta o la gestione del team. ${ }^{10}$

Come manager, una delle responsabilità più importanti degli infermieri è la formazione sanitaria continua, poiché ha conoscenze teoriche e scientifiche. Questa educazione sanitaria è tradotta più regolarmente dalle linee guida e dalle spiegazioni trasmesse alla famiglia della vittima che correttamente ai dipendenti infermieristici, dove gli infermieri sono responsabili della formazione e della formazione dei loro dipendenti, mirando a migliore qualità dei servizi forniti ${ }^{1}, 10$

\section{CONCLUSIONE}

Dei 9 articoli individuati per raggiungere gli obiettivi proposti, il 100\% (n.9) si è rivolto alle competenze di assistenza, ma di questo totale, solo il $33 \%$ (n. 3) si è rivolto alle competenze gestionali della categoria infermieristica. Per le competenze gestionali, quelli che si sono distinti sono quelli che hanno un rapporto diretto con la vittima, come la fornitura di assistenza. Mentre i manager hanno trovato competenze che non sono 
direttamente collegate alla vittima, con attività amministrative come le principali, come il dimensionamento del team. Si conclude che gli infermieri sono un professionista indispensabile sia nella cura diretta della vittima che nelle azioni che coordinano questa cura.

\section{RIFERIMENTI}

1. LUCHTEMBERG, Marilene Nonnemacher; PIRES, Denise Elvira Pires de. Enfermeros del Servicio de Atenciàn M'vil de Urgencia: profilo y desarrolladas attività. Giornale brasiliano di infermieristica, v. 69, n. 2, p. 213220, 2016.

2. Adamo, Rodrigo de Souza; SANTOS, Maria Regina dos. Prestazioni degli infermieri nell'assistenza mobile pre-ospedaliera. Revista Mineira de Enfermagem, v. 16, n. 4, p. 601-608, 2012.

3. BERNARDES, Andrea ealtri. Supervisione delle infermiere nell'assistenza mobile pre-ospedaliera. Giornale infermieristico elettronico, v. 16, n. 3, pa. 635-43, 2014.

4. BRASILE, Ministero della Salute. Politica nazionale per l'assistenza di emergenza. Brasilia, redattore del Ministero della Salute, 3. ed. ampl., 2006.

5. COSTA, Gabriela. Un approccio all'azione della storia infermieristica di fronte alle politiche sanitarie. Revista Mineira de Enfermagem - REME; vol 10.4; Joào Pessoa - PB, 2016

6. EL HETTI, Livia Barrionuevo et al. Formazione continua/continua come strategie di gestione nel servizio mobile di emergenza. Giornale infermieristico elettronico, v. 15, n. 4, p. 973-82, 2013.

7. MENDES, Euginio Vilaa. Reti sanitarie. Cinc. salute collettiva, Rio de Janeiro, v. 15, n. 5, p. 2297-2305, agosto 2010.

8. MESQUITA, Kayena Lopes et al. II punto di vista dell'infermiere/manager sulla necessità di implementare il supporto psicologico ai professionisti del servizio di assistenza mobile di emergenza. Giornale infermieristico del Midwest Di Minas Gerais, 2014. 
9. NETTINA, Sandra ( Pratica infermieristica. 9ed Ed. Rio de Janeiro: Guanabara Koogan, 2011.

10. PERES, Paulo Sergio Quevedo et al. Prestazioni degli infermieri in un servizio di assistenza pre-ospedaliera privato/Nurse prestazioni su un'assistenza preospedaliera privata. Rivista di ricerca: La cautela è fondamentale online, v. 10, n. 2, p. 413-422, 2018.

11. TAVARES, Tayrine Ypuena et al. La vita quotidiana degli infermieri che lavorano nel servizio di assistenza mobile di emergenza. Giornale infermieristico del Midwest di Minas Gerais, v. 7, 2017.

Inviato: Novembre, 2019.

Approvato: novembre 2019. 